\title{
SAPHO Syndrome in an Adult Male with Ulcerative Colitis
}

\author{
Sana Sayeed, Areeba Imtiaz and Faiza Naz
}

\begin{abstract}
Synovitis Acne Pustulosis Hyperostosis Osteitis (SAPHO) syndrome refers to a rare entity expressed by a combination of dermatological and osteoarticular manifestations. We report a case of this unique syndrome initially diagnosed as "Sweet's syndrome" due to more or less similar features. The patient is also a diagnosed case of ulcerative colitis, a rare association with SAPHO syndrome. The clinical picture, laboratory findings and most of all radiological features, ultimately directed us towards the final diagnosis. The purpose of this case report is to raise awareness regarding this unusual syndrome, its clinical manifestations, laboratory and radiological findings, so that proper management can be carried out in time.
\end{abstract}

Key Words: SAPHO syndrome, Dermatological, Osteoarticular, Sweet's syndrome, Ulcerative colitis.

\section{INTRODUCTION}

SAPHO, the acronym first introduced by a group of French researchers (Chamot et al.), is contemplated to be a type of seronegative spondyloarthropathy. 1 The disease may present at any age, however, children and young to middle-aged adults are most commonly affected. 2 In most of the literature, the prevalence of this syndrome is stated to be $<1 / 10000$, which may be an underestimate, due to its inaccurate diagnosis. 2,3 The anterior chest wall is the most commonly involved site. ${ }^{4}$ SAPHO syndrome is rarely associated with inflammatory bowel disease, such as ulcerative colitis and Crohn's disease. Similarly, patients with inflammatory bowel disease rarely develop this syndrome.5,6 Awareness of this syndrome is necessary to differentiate it from other similar conditions to prevent or avoid its misdiagnosis.1,7

We present a case of a young male from North Pakistan, a diagnosed case of ulcerative colitis, who developed skin, osseous and articular features of SAPHO syndrome gradually with the involvement of anterior chest wall. We intend to highlight the diagnostic challenges faced in such cases.

\section{CASE REPORT}

We encountered this infrequent syndrome in a 31-year male patient from North Pakistan. The patient was a diagnosed case of ulcerative colitis for one year which was biopsy-proven. He was doing relatively well until about three months back when small papule like lesions started appearing on his back, arms and legs. He also developed a large ulcerating lesion on his right forearm.

Department of Radiology, Shifa International Hospital,

Islamabad, Pakistan

Correspondence: Dr. Areeba Imtiaz, House No. 404/A,

Street 4, Corniche Road, Spring North, Bahria Town, Phase 7,

Rawalpindi, Pakistan

E-mail: areeba_mahera@hotmail.com

Received: August 09, 2018; Revised: November 18, 2018;

Accepted: November 19, 2018
There was recent emergence of two tender swellings over his sternum with pain in costochondral joints. History of weight loss was also positive. The skin changes were diagnosed initially as chronic dermatitis. The clinical findings and history initially led the physicians to make a diagnosis of "Sweet's syndrome" (acute febrile neutrophilic dermatosis). Multiple investigations were carried out which showed raised C-reactive protein (CRP) and erythrocyte sedimentation rate (ESR). There was slightly raised total leukocyte count (TLC). Rest of the complete blood picture, liver function tests, renal function tests, calcium and phosphorus levels were within normal limits. Skin biopsy showed variable dermal edema with mixed perivascular dermal infiltrate including neutrophils. Antinuclear antibody (ANA), centrally accentuated antineutrophil cytoplasmic antibody (c-ANCA), perinuclear anti-neutrophil cytoplasmic antibodies (P-ANCA) and human immunodeficiency virus (HIV) antibody were nonreactive. His angiotensin converting enzyme (ACE) and lactate dehydrogenase (LDH) levels were also normal. Entamoeba histolytica was positive on stool routine examination. His sternal swellings progressed in size. Chest radiograph showed multiple, ill-defined lucent areas in the sternum. Contrast enhanced computed tomography (CT) chest, abdomen and pelvis was performed for further evaluation of the chest wall lesions and to see the extent of bowel disease. It showed marginally enhancing hypodense collections/abscesses along the entire sternal body and right lateral aspect of manubrium sterni, cortical irregularity, ill-defined lucencies and patchy sclerosis suggestive of osteitis, few air specks in the subcutaneous tissue/skin in the anterior abdo-minal wall possibly representing pustules and colitis involving left-sided colon from splenic flexure till rectum (Figures 1, 2, 3). Clinical correlation for SAPHO syndrome was suggested. Patient was started on intravenous (IV) antibiotics and corticosteroids. His CRP and ESR levels started improving. Patient was discharged about one week later, when he became vitally stable with much improvement in gastrointestinal 


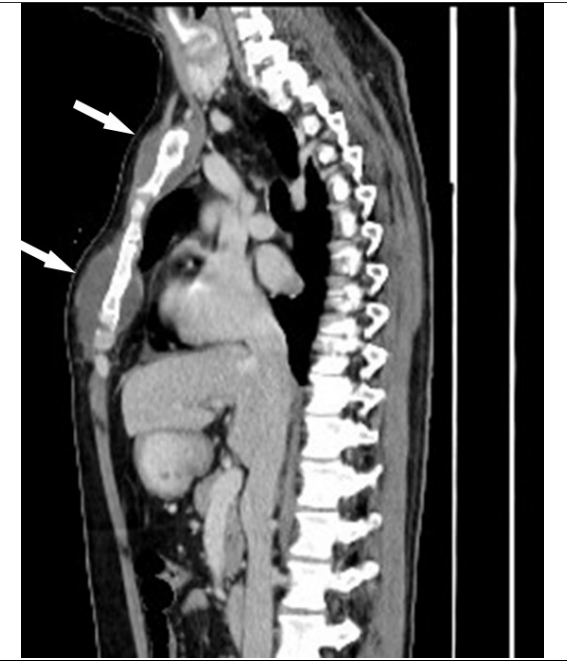

Figure 1: Marginally enhancing hypodense collections/abscesses along the sternal body and manubrium sterni (arrows), projecting into the anterior mediastinum

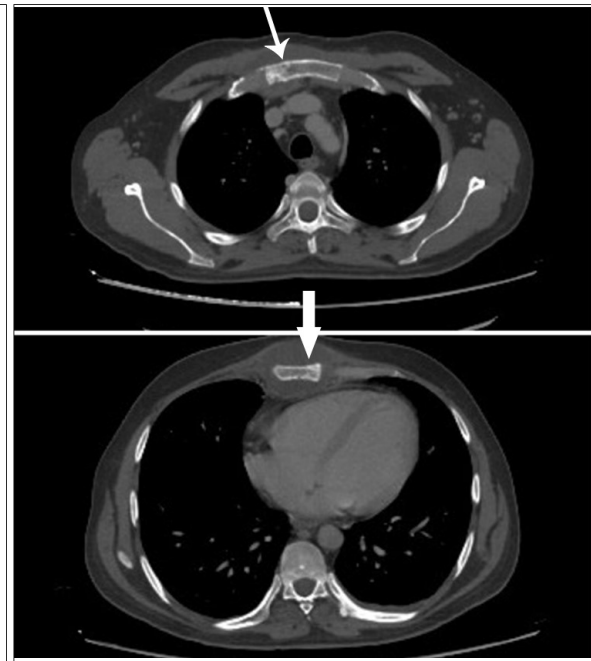

Figure 2: Subchondral erosive changes with cortical irregularity, ill-defined lucencies and patchy sclerosis involving manubrium sterni (thin arrow) and body of sternum (thick arrow), representative of osteitis/osteomyelitis.

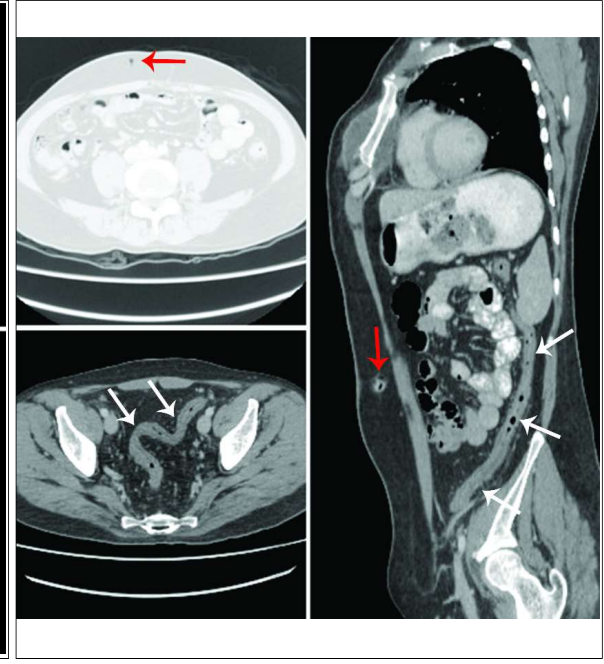

Figure 3: Diffusely thick walled and ahuastral left hemi-colon from splenic flexure till rectum with accentuated mucosal enhancement and para-colic congestive and strandy changes, suggesting colitis (white arrows). Few air specks in subcutaneous fat of right anterior abdominal wall, likely pustulosis (red arrows). symptoms as well as cutaneous changes. He was continued on IV antibiotics and oral corticosteroids for six weeks and on regular follow-up. His sternal lesions have resolved, CRP is negative, ESR and TLC are within normal limits. He is currently on oral corticosteroids for symptomatic relief and underwent colonoscopy with repeat biopsy at our facility due to recurrence of diarrhea as previous biopsy was done from outside. The latest tissue sampling showed left sided ulcerative colitis without evidence of malignancy.

\section{DISCUSSION}

This peculiar syndrome of bones, joints and skin abnormality, known by various (more than 50) names in the past, has been very popular in Western Europe and Japan with multiple publications in last 10 years. ${ }^{1}$ It can present at any age; however, most commonly it is seen in children and young to middle-aged adults without any specific preponderance for gender. 2

There is still poor understanding about the pathophysiology of this entity. Three different hypotheses, namely molecular mimicry, immune complex and immune barrier breakdown hypotheses have been proposed. Possibility of genetic predisposition with immunological response to causative agent, Propionibacterium acnes, identified in some of the cutaneous lesions, is also described in the literature.1,2,4

Recurrences and remissions are usually observed in the clinical course of this syndrome, which is usually selflimiting. 4 The dermatological and osteoarticular phenomenon may not occur simultaneously. ${ }^{1}$ The diagnosis warrants the presence of at least one out of the four criteria, including osteoarticular features with severe acne, palmoplantar pustulosis with osteoarticular features, hyperostosis with or without dermatosis and lastly multifocal recurrent chronic osteomyelitis of the axial or appendicular skeleton, with or without skin lesions. ${ }^{3}$ The site most frequently involved is the anterior chest wall (65-90\%), followed by spine (thoracic, lumbar >cervical), pelvis and lastly the long bones.3,4 This patient also presented with skin manifestations preceding the involvement of sternum and sterno-clavicular joints.

Laboratory investigations do not have much role in the diagnosis of SAPHO syndrome; however, there might be signs of inflammation, raised CRP and ESR as well as mildly raised TLC,1 as was initially seen in our case. Bone biopsy may prove helpful, if the presentation is unusual. ${ }^{1}$ Radiology plays a crucial role in its early diagnosis. Radiographs, bone scintigraphy, CT and magnetic resonance imaging $(\mathrm{MRI})$ are of importance with $\mathrm{CT}$ being sensitive in detecting bony abnormalities such as hyperostosis and sclerosis. 3,6 Osseous and soft tissue edema as well as involvement of spine, intervertebral discs and synovitis can be picked on MRI.3,4,6 In this case, CT findings led to the diagnosis of SAPHO syndrome when correlated with history of the patient, clinical picture and laboratory findings.

One of the reasons of underdiagnosis of SAPHO syndrome is the resemblance between its features and those of the extra intestinal manifestations of inflammatory bowel disease with which it is rarely associated. 6 As described before, patient in our case was also suffering from ulcerative colitis, a known association of SAPHO syndrome. A case of enteropathic SAPHO syndrome was presented by Siau et al. in 2009 describing a 39-year female with ulcerative colitis, later 
on diagnosed as having SAPHO syndrome as well; primarily involving the right clavicle and showing good response to pamidronate therapy. 6 In another study performed by Naves et al., three patients were presented having coexistent SAPHO syndrome and inflammatory bowel disease among the 62 patients with SAPHO syndrome $(4.8 \%))^{5}$

At present, there is no specific treatment for SAPHO syndrome; however, symptomatic relief with first-line agents including non-steroidal anti-inflammatory drugs and corticosteroids is the main objective.3,5-7 Antibiotics have also been tried considering the detection of propionibacterium acnes in bone biopsies of few patients without any definite promising results.5,7 Recently, inclusion of drugs like methotrexate, intra-venous pamidronate, calcitonin, bisphosphonates and antitumor necrosis factor agents in the management of this syndrome has shown reassuring results. ${ }^{1,7}$

To conclude, different modalities of radiology along with clinical manifestations play a vital role in the challenging diagnosis of this syndrome affecting skin, bones and joints having overlapping features with multiple other conditions.

\section{REFERENCES}

1. Boutin, R, Resnick D. The SAPHO syndrome: An evolving concept for unifying several idiopathic disorders of bone and skin. AJR Am J Roentgenol 1998; 170:585-91.

2. Nguyen M, Borchers A, Selmi C, Naguwa S, Cheema G, Gershwin M. The SAPHO syndrome. Seminars in Arthritis and Rheumatism 2012; 42:254-65.

3. Duan N, Chen X, Liu Y, Wang J, Wang Z. Multimodal imaging findings of SAPHO syndrome with no skin lesions: A report of three cases and review of the literature. Exp Ther Med 2016; 12:2665-70.

4. Tohme-Noun C, Feydy A, Belmatoug N, Krainik A, Fantin B, Menu Y. Cervical involvement in SAPHO syndrome: Imaging findings with a 10-year follow-up. Skeletal Radiol 2003; 32:103-6.

5. Naves J, Cabré E, Mañosa M, Grados D, Olivé A, Domènech E. A systematic review of SAPHO syndrome and inflammatory bowel disease association. Dig Dis Sci 2013; 58:2138-47

6. Siau K, Laversuch CJ. SAPHO syndrome in an adult with ulcerative colitis responsive to intravenous pamidronate: A case report and review of the literature. Rheumatol Int 2010; 30:1085-8

7. Gorecki P, Stockmann P, Distler J, Wuest W, Schmidt D, Neukam $F$, et al. Implication of bisphosphonate use in the treatment of SAPHO syndrome: Case report and discussion of current literature. J Med Hypotheses Ideas 2015; 9:72-8. 